\title{
CARACTERISATION PHYSICO-CHIMIQUE ET MICROBIOLOGIQUE DES BOUES DE VIDANGE DESHYDRATEES ET DES DECHETS SOLIDES MENAGERS ORGANIQUES POUR UNE VALORISATION EN COMPOST AGRICOLE
}

\author{
Soré Ouindpouiré Auguste A. ${ }^{1, ~ *}$, and Ouoba Samuel ${ }^{1, * *}$ \\ ${ }^{1}$ Laboratoire de Physique et de Chimie de l'Environnement, Université Joseph KI-ZERBO, \\ Ouagadougou, Burkina Faso, \\ *Email: auguste.sore@gmail.com \\ **Email : samuelouoba1@yahoo.fr
}

INFOS SUR L'A R T I C L E

Historique de l'article:

Reçu le : 17 novembre 2020

Reçu en format revisé le : 07 novembre 2021

Accepté le : 10 novembre 2021

Mots-Clés : Boues de vidange

déshydratées, Déchets solides

ménagers organiques, Compostage

Keywords : Dewatered faecal sludge, Organic household solid waste, Composting
RE S U M E

Dans la ville de Ouagadougou au Burkina Faso, les stations de traitement des boues de vidange souffrent du manque d'une filière de valorisation des boues après séchage. Pour les déchets solides ménagers, les quantités collectées sont majoritairement destinées à l'enfouissement technique. Trouver une solution pour la valorisation des boues de vidange déshydratées (BVD) et des déchets solides ménagers organiques (DSMO) est nécessaire en vue de compléter les filières «gestion des boues de vidange » et « gestion des déchets solides ». Notre travail vise à faire une étude physico-chimique, microbiologique et éco-toxicologique d'un compost à base de BVD et de DSMO afin de proposer une approche méthodologique pour la production durable d'un compost hygiénique. Cet article aborde la première étape de notre recherche qui a consisté à caractériser les BVD à la station de traitement de Zagtouli ainsi que les DSMO du centre de traitement et de valorisation des déchets de la ville de Ouagadougou, afin d'identifier une filière de valorisation adaptée. Pour ce faire, un échantillon représentatif a été prélevé sur le tas des BVD de Zagtouli pour analyse au laboratoire. L'échantillon de DSMO a été obtenu à partir de bacs d'ordures ménagères provenant des centres de pré-collecte de la ville de Ouagadougou. Les caractéristiques brutes des BVD et des DSMO (matière organique, azote, $\mathrm{pH} .$. ) et leur valeur en éléments minéraux (calcium, magnésium, potassium, phosphore) montrent que ces substrats seraient idéals pour la production d'un compost de qualité.

\footnotetext{
A B S T R A C T

In the city of Ouagadougou in Burkina Faso, faecal sludge treatment plants suffer from the lack of a process for recovering sludge after drying. For solid household waste, the quantities collected are mainly intended for technical landfill. Finding a solution for the recovery of Dewatered Faecal Sludge (DFS) and Organic Household Solid Waste (OHSW) is necessary in order to complete the faecal sludge management and solid waste management sectors. Our work aims to make a physico-chemical, microbiological and eco-toxicological study of a compost based on DFS and OHSW in order to propose a methodological approach for the sustainable production of hygienic compost. This article discusses the first step of our research, which consisted in characterizing the DFS at the Zagtouli treatment station as well as the OHSW of waste treatment and recovery center of Ouagadougou in order to identify a suitable recovery channel. To do so, a representative sample was taken from the Zagtouli DFS heap for laboratory analysis. The OHSW sample was obtained from household garbage bins from pre-collection centers in the city of Ouagadougou. The raw characteristics of DFS and OHSW (organic matter, nitrogen, $\mathrm{pH}$, etc.) and their value in mineral elements (calcium, magnesium, potassium, phosphorus) show that these substrates would be ideal for the production of quality compost.
} 


\section{INTRODUCTION}

La problématique de la gestion des boues de vidange dans les pays en voie de développement demeure un défi à relever à cause du déversement anarchique des boues dans l'environnement et leur utilisation de façon non hygiénique dans l'agriculture (Zakaria et Fiona, 2019). Dans la ville de Ouagadougou, capitale du Burkina Faso, plus de $95 \%$ de la population a recours à des ouvrages d'assainissement autonome pour la gestion des eaux usées et excrétas (Bassan et al. 2012). Les quantités de boues de vidange produites sont donc croissantes.

Pour limiter les conséquences liées à la mauvaise gestion des boues vidange, le Burkina a construit, surtout dans les grandes villes, des stations de traitement. La principale difficulté actuelle de ces stations demeure la valorisation des sous-produits issus du traitement, notamment les BVD. Kone et al., (2016) suggèrent le compostage comme filière de valorisation des boues de vidange traitées par séchage dans les stations à Ouagadougou. Pour améliorer la qualité du compost produit à base de BVD, Cofie et al., (2009) proposent un co-compostage avec les DSMO.

La qualité d'un compost dépend grandement des substrats à utiliser pour le compostage (Lo et al. 2019). Il est donc nécessaire, avant d'entamer le processus de cocompostage, de faire une caractérisation des BVD ainsi que des DSMO (Cofie et al. 2009). Cette caractérisation qui décrit les potentialités des substrats de compostage donne également une idée sur la qualité du compost à obtenir.

La Station de Zagtouli, mis en service depuis 2014 déverse jusque-là ses BVD à l'air libre. Ces BVD ainsi gérées pourraient représenter des risques pour les populations et l'environnement (Zakaria et Fiona, 2019). Cette station a été identifiée comme site de prélèvement des BVD à caractériser. Les DSMO ont été obtenus à partir des bacs d'ordures en provenance des centres de pré-collecte de la ville de Ouagadougou.

Cette étude vise (i) à déterminer les caractéristiques physico-chimiques des BVD et DSMO de la ville de Ouagadougou, (ii) à connaître la qualité hygiénique de ces substrats et enfin (iii) à apprécier la qualité de ces substrats en vue d'une valorisation en compost agricole.

À terme, c'est un travail qui va contribuer à une meilleure gestion de la filière des boues de vidange et des déchets solides dans la ville de Ouagadougou.

\section{MATERIEL ET METHODES}

\section{1 Échantillonnage des BVD et des DSMO}

L'échantillon de BVD a été prélevé à la station de traitement de Zagtouli. Pour ce faire, la méthode « d'échantillonnage dans un tas » proposée par Chabalier et al. (2006) a été utilisé. L'échantillon a été constitué à partir de sous-échantillons qui ont été pris suivant trois techniques de prélèvement : un prélèvement en diagonal, un prélèvement en zigzag et un prélèvement aléatoire. Chaque technique a permis de prélever sur dix (10) points du tas. Sur chaque point, le prélèvement a été fait audessus, au milieu et au fond du tas. Le mélange de ces souséchantillons a constitué l'échantillon représentatif du tas de BVD. A partir de cet échantillon représentatif, un échantillon élémentaire à analyser au laboratoire a été constitué en utilisant la méthode des «1/4» proposée par Cugnon et Lambert (2017).

Les DSMO à caractérisés ont été obtenus à partir des déchets solides des bacs d'ordures ménagères provenant des centres de pré-collecte de la ville de Ouagadougou. Un tri manuel de ces déchets a été réalisé afin de séparer la partie biodégradable, notamment les fermentescibles alimentaires (FA), les déchets de jardin (DJ) et les papierscartons (PC). Ces différentes catégories ont été ensuite mélangées dans les proportions des sous-catégories des déchets organiques de la ville de Ouagadougou indiquées dans le tableau 1 (Haro et al., 2018). La technique des « 1/4 » de Lambert et Cugnon (2017) a été enfin utilisée pour prélever l'échantillon élémentaire à analyser au laboratoire.

Tableau 1 : Composition moyenne par sous-catégories des DSMO de la ville de Ouagadougou (Haro, 2018)

\begin{tabular}{|l|c|}
\hline Sous-Catégories & Quantité (\%) \\
\hline Fermentescibles Alimentaires (FA) & 12,46 \\
\hline Déchets de Jardin (DJ) & 9,47 \\
\hline Papier/Carton (PC) & 6,83 \\
\hline
\end{tabular}

\subsection{Analyses physico-chimiques}

Le $\mathrm{pH}$ et la conductivité électrique (CE) ont été mesurés à l'aide d'un multi-paramètre HANNA HI 4222. La teneur en eau (W) et la matière organique (MO) ont été mesurées respectivement par passage à l'étuve à $105^{\circ} \mathrm{C}$ pendant $24 \mathrm{~h}$ et au four à $550^{\circ} \mathrm{C}$ pendant $2 \mathrm{~h}$. Le carbone organique total (COT) a été déduit de la matière organique en appliquant le facteur 1/2 de Giroux et Audesse (2004). La teneur en azote total Kjeldhal (NTK) a été mesurée après minéralisation, distillation et titrimétrie. L'azote ammoniacal $\left(\mathrm{N}-\mathrm{NH}_{4}{ }^{+}\right)$et l'azote nitrique $\left(\mathrm{N}-\mathrm{NO}_{3}{ }^{-}\right)$ont été déterminés par un spectrophotomètre d'absorption moléculaire (DR 2000). L'azote organique a été déduit par la différence entre le NTK et le N-NH${ }_{4}^{+}$. L'azote total a été déterminé en faisant la somme des différentes formes d'azote déjà mesurées $\left(\mathrm{NTK}, \mathrm{N}-\mathrm{NO}_{3}{ }^{-}, \mathrm{N}-\mathrm{NO}_{2}{ }^{-}\right)$.

Les concentrations en fertilisants majeurs (phosphore et potassium) et en fertilisants mineurs (calcium et magnésium) ont été également évaluées. Le phosphore (P) a été déterminé par un spectrophotomètre d'absorption moléculaire DR 2000 après minéralisation. Le potassium (K) a été mesuré avec un multi-paramètre HANNA HI 4222. Le calcium $(\mathrm{Ca})$ et le magnésium $(\mathrm{Mg})$ ont été déterminés par volumétrie suivi d'un titrage à l'EDTA. 


\subsection{Analyses microbiologiques}

Les indicateurs de contamination fécale, notamment les coliformes fécaux (CF) et les streptocoques fécaux (SF), ont été déterminés par la méthode Apha (1998) respectivement sur les milieux de culture Chromocult Agar pour coliformes et Chromocult Agar pour streptocoques. La charge parasitaire à travers les œufs d'helminthes a été déterminée par la méthode de US-EPA (1999), modifié par Schwartzbrod J. et al (2003).

\subsection{Traitement des données}

Les résultats de cette étude sont des moyennes obtenues à partir des mesures répétées. Les comparaisons ont été faites en utilisant le test $\mathrm{t}$-student XLS Stat.

\section{RESULATS ET DISCUSSION}

\subsection{Caractéristiques physico-chimiques des BVD et des} DSMO

Les caractéristiques physico-chimiques des substrats BVD et DSMO sont présentés dans le tableau 2 et montrent une grande variabilité. Le $\mathrm{pH}$ varie au tour de la neutralité avec des valeurs de 6,2 et de 7,85 respectivement pour les BVD et les DSMO. La teneur en eau est faible dans les BVD (34\%) comme dans les DSMO (20\%). Les deux substrats ont également une bonne teneur en MO $(25,62 \%$ et de $58,42 \%$ respectivement pour les BVD et les DSMO). La teneur en azote NTK dans les BVD est légèrement faible $(1,22)$ comparée à la teneur $(1,67)$ dans les DSMO. Les valeurs des rapports $\mathrm{C} / \mathrm{N}$ restent élevées pour les deux substrats $(18,66$ et 28,03 respectivement pour les BVD et les DSMO). La présence de minéraux tels que le calcium, le magnésium, le potassium ainsi que le phosphore en teneur appréciable est également remarquable dans les deux (02) substrats.

Tableau 2 : Caractéristiques physico-chimiques des substrats BVD et DSMO

\begin{tabular}{|r|c|c|c|}
\hline Paramètres & Unités & BVD & DSMO \\
\hline $\mathbf{p H}$ & & $6,12 \pm 0,01$ & $7,85 \pm 0,01$ \\
\hline $\mathbf{C E}$ & $\mathrm{mS.cm}{ }^{-1}$ & $3,50 \pm 0,25$ & $5,25 \pm 0,75$ \\
\hline $\begin{array}{r}\text { Teneur en } \\
\text { Eau }\end{array}$ & $\%$ & $34,00 \pm 2$ & $20,00 \pm 3$ \\
\hline $\begin{array}{r}\text { Matière } \\
\text { Organique } \\
\text { (MO) }\end{array}$ & $\%$ & $25,62 \pm 2,59$ & $58,42 \pm 5,1$ \\
\hline Carbone C & $\%$ & $12,81 \pm 1,29$ & $29,21 \pm 2,55$ \\
\hline NTK & $\%$ & $1,22 \pm 0,32$ & $1,67 \pm 0,08$ \\
\hline N-NH4 & $\%$ & $0,43 \pm 0,05$ & $0,61 \pm 0,02$ \\
\hline N-NO3 & $\%$ & $0,47 \pm 0,06$ & $2,76 \pm 0,79$ \\
\hline Norg & $\%$ & $0,79 \pm 0,27$ & $1,06 \pm 0,1$ \\
\hline $\mathbf{C} / \mathbf{N}$ & & $18,99 \pm 8,12$ & $28,03 \pm 5,05$ \\
\hline Potassium K & $\%$ & $0,74 \pm 0,03$ & $4,97 \pm 0,35$ \\
\hline Calcium Ca & $\%$ & $0,36 \pm 0,07$ & $0,39 \pm 0,04$ \\
\hline Magnésium & $\%$ & $0,16 \pm 0,02$ & $0,17 \pm 0,03$ \\
\hline Mg & $\%$ & $4,07 \pm 1,12$ & $5,30 \pm 0,55$ \\
\hline Phosphore P & $\%$ & & \\
\hline & & & \\
\hline
\end{tabular}

2.2 Caractéristiques microbiologiques des BVD et DSMO

Les caractéristiques microbiologiques des substrats BVD et DSMO sont présentés dans le tableau 3. Les CF notamment des E. Coli sont très abondants dans les BVD $\left(1,20 \mathrm{E}+03\right.$ UFC.g ${ }^{-1}$ pour les CF et $3,99 \mathrm{E}+02$ UFC. $\mathrm{g}^{-1}$ pour E. Coli), par contre ils sont en nombre réduit dans les DSMO (76,00 UFC. $\mathrm{g}^{-1}$ pour les CF et 25,50 UFC.g ${ }^{-1}$ pour les E. Coli). Ces deux substrats ont une charge relativement faible en SF, soient $37,00 \mathrm{UFC} \cdot \mathrm{g}^{-1}$ pour les BVD et 4,50 UFC. $\mathrm{g}^{-1}$ pour les DSMO. Les œufs d'helminthes sont présents dans les BVD (21,00 œufs. $\left.\mathrm{g}^{-1}\right)$ par contre les DSMO sont exempts d'œufs d'helminthes.

Tableau 3 : Caractéristiques microbiologiques des substrats BVD et DSMO

\begin{tabular}{|c|c|c|c|}
\hline \multicolumn{4}{|c}{ substrats $B V D$ et $D S M O$} \\
\hline $\begin{array}{c}\text { Paramètres } \\
\text { Fécaux CF }\end{array}$ & Unités & BVC.g & B \\
\hline E. coli & $\begin{array}{c}1,20 \mathrm{E}+03 \pm \\
3,47 \mathrm{E}+02\end{array}$ & $76,00 \pm 19$ \\
\hline $\begin{array}{c}\text { Streptocoques } \\
\text { Fécaux SF }\end{array}$ & UFC.g $^{-1}$ & $\begin{array}{c}3,99 \mathrm{E}+02 \pm \\
1,16 \mathrm{E}+02\end{array}$ & $25,50 \pm 6,5$ \\
\hline $\begin{array}{c}\text { OEufs } \\
\text { d'helminthes }\end{array}$ & œufs.g ${ }^{-1}$ & $21,00 \pm 2$ & 0 \\
\hline
\end{tabular}

\subsection{Discussion}

Le $\mathrm{pH}$ des BVD et des DSMO, variant autour de la neutralité, montre que ces substrats constitueraient un milieu favorable pour la prolifération des bactéries responsables de la dégradation de la MO au cours d'un compostage. Ces substrats ont une bonne teneur en matières organiques. Ceci pourrait permettre un développement et une prolifération rapide des populations de bactéries et de champignons au cours d'un processus de compostage (Chennaoui et al., 2016). La teneur en azote NTK dans les BVD qui est légèrement faible par rapport à celle dans les DSMO est due à une perte d'azote au cours de la déshydratation lors du traitement des BV (Kone et al. 2016). Les valeurs des rapports $\mathrm{C} / \mathrm{N}$ qui sont largement supérieurs à 15 pour les deux substrats montrent qu'il faut au préalable soumettre les BVD et les DSMO à un processus de minéralisation si on veut les utiliser comme fertilisants agricoles (Lo et al., 2019). Pour des tests de compostage, un choix des rapports de mélange en volume de DSMO/BVD pourrait être faite de sorte à obtenir un rapport $\mathrm{C} / \mathrm{N}$ compris entre 20 et 30 pour permettre un bon déroulement du processus de compostage (Diaz et al. 2011). Les bonnes teneurs en fertilisants majeurs (N, P et $\mathrm{K}$ ) et en fertilisants mineurs ( $\mathrm{Ca}$ et $\mathrm{Mg}$ ) prouvent la qualité agronomique de ces substrats. En somme, Les caractéristiques physico-chimiques des BVD et des DSMO montrent que ces substrats sont idéals pour la production d'un compost de qualité (Cofie et al., 2009).

Les caractéristiques microbiologiques des BVD confirment qu'après séchage, les BVD restent des déchets 
dangereux pour l'homme et pour l'environnement (Talon, 2014). La charge en CF et en E. Coli des BVD témoigne d'une présence très probable de pathogènes dans ces substrats. La présence des œufs d'helminthes confirme que l'utilisation directe des BVD en agriculture devrait être proscrite (Zakaria et Fiona, 2019). Les BVD devraient donc être soit directement mises en décharge, soit soumises à un traitement d'hygiénisation si une valorisation agricole est envisagée (Mbéguéré et al., 2011). Tenant compte des réalités socio-économiques du Burkina Faso, le compostage serait une filière d'hygiénisation adaptée pour la valorisation des BVD (Strande et al., 2018). L'analyse des caractéristiques microbiologiques des DSMO montre qu'ils sont moins dangereux que les BVD. Une combinaison des DSMO avec les BVD pourrait permettre d'avoir des conditions optimales pour la croissance des micro-organismes qui aideraient à obtenir un compost de qualité améliorée (Sánchez et al. 2015).

\section{CONCLUSION ET PERSPECTIVES}

Cette étude a pour objectif d'évaluer la faisabilité d'un compostage à base des BVD de la station de Zagtouli et des DSMO de la ville de Ouagadougou. La caractérisation physico-chimique a montré les potentialités des BVD et les DSMO qui font d'eux des substrats idéals pour le compostage. La caractérisation microbiologique a montré qu'il est impératif de soumettre ces substrats, notamment les BVD, à un processus d'hygiénisation avant d'autoriser leur utilisation en agriculture. En considérant les réalités socio-économiques du Burkina, le compostage s'avère être une filière simple, non couteuse et pratique pour la valorisation des BVD et des DSMO.

Pour aller plus loin dans cette réflexion, il serait important de faire un suivi des essais de co-compostage pour déterminer un ratio optimal en volume de mélange de DSMO/BVD et le temps de compostage nécessaire pour obtenir un compost stable et mature.

\section{REFERENCES}

Apha, A. (1998). Standard methods for the examination of water and wastewater, 20th ed. American Public Health Association, Washington.

Bassan M., Tchonda T., Mbeguere M., Strande L. (2012). Optimizing the faecal sludge management scheme in Ouagadougou, Burkina Faso. Sustainable Sanitation Practice, vol. 13, pp. 22-24.

Chabalier, P., Van de Kerchove, V., \& Saint Macary, H. (2006). Guide de la fertilisation organique à La Réunion. CIRAD, France.

Chennaoui, M., Salama, Y., Aouinty, B., (2016). Impact de l'evolution de la flore microbienne sur les variations des paramètres physico-chimiques lors du compostage en cuve des déchets ménagers. Revue des Bioressources, vol. 6, n².
Cofie O., Kone D., Rothenberger S., Moser D., Zubruegg C., (2009). Co-composting of faecal sludge and organic solid waste for agriculture: Process dynamics. Water research, vol. 43, n 18 , pp. 4665-4675.

Cugnon, T., Lambert, R., \& Mahillon, J. (2017). Corrélation entre le potentiel de minéralisation et la quantité d'azote réellement minéralisé sur trois parcelles de prairies cultivées. In 13èmes Rencontres de la fertilisation raisonnée et de l'analyse.

Diaz L., De Bertoldi M., Bidlingmaier W. (2011). Science et technologie du compost. Elsevier, Amsterdam.

Giroux, M. Et Audesse, P., (2004). Comparaison de deux méthodes de détermination des teneurs en carbone organique, en azote total et du rapport $\mathrm{C} / \mathrm{N}$ de divers amendements organiques et engrais de ferme. Agrosol, vol. 15, n², pp. 107-110.

Joceline S., Kone M., Yacouba O., Arsène Y. (2016). Planted sludge drying beds in treatment of faecal sludge from Ouagadougou: Case of two local plant species. Journal of Water Resource and Protection, vol. 8, n07, pp. 697.

Kone M., Ouattara Y., Ouattara P., Bonou L., Joly P. (2016). Caractérisation des boues de vidange dépotées sur les lits de séchage de zagtouli (Ouagadougou). International Journal of Biological and Chemical Sciences, vol. 10, n6, pp. 2781-2795.

Lo, M., Dieng, D., Ndiaye, S., Diop, C., Seck, A., Gueye, A. (2019). Co-compostage de boues de vidange domestiques avec des déchets maraîchers et des déchets de poissons à Dakar (Sénégal). International Journal of Biological and Chemical Sciences, vol. 13, n6, pp. 2914-2929.

Mbéguéré, M., Dodane, P. H., \& Koné, D. (2011). Gestion des Boues de Vidange-optimisation de la filière. Eawag, Dübendorf, Suisse.

Sánchez, Antoni, Gabarrell, Xavier, Artola, Adriana, (2015). Composting of wastes. Resource Recovery to Approach Zero Municipal Waste. Green Chemistry and Chemical Engineering Series. CRC Press, Taylor \& Francis Group, pp. 77-106.

Schwartzbrod J., Gaspard P. (1998). Quantification and viability determination for helminth eggs in sludge (Modified EPA France, 1993 Method). Faculty of Pharmacy, University "Henri Poincare" Department of Microbiology, France.

Strande L., Ronteltap M., Brdjanovic D. (2018). Gestion des Boues de Vidange : Approche intégrée pour la mise en œuvre et l'exploitation. IWA Publishing, Fance.

Zakaria F. (2019). Rethinking Faecal Sludge Management in Emergency Settings: Decision Support Tools and Smart Technology Applications for Emergency Sanitation. CRC Press, London. 